\title{
On the Harish-Chandra Condition for First-Order Relativistically-Invariant Free Field Equations
}

\author{
A. S. Glass* \\ Joseph Henry Laboratories of Physics, Princeton University, Princeton, New Jersey, USA
}

Received June 1, 1971

\begin{abstract}
It has hitherto been accepted that the degree of the Harish-Chandra condition applying to single-mass equations of arbitrary spin is determined by the maximum spin appearing in the representation of $S L(2, C)$ which acts on the field. The present paper demonstrates a fallacy in the published arguments which lead to the above conclusion, and gives the correct conclusion which can be deduced from the hypotheses. A counterexample of an irreducible, single-mass, spin $3 / 2$ equation which does not satisfy the accepted theory is provided in an appendix.
\end{abstract}

Relativistically-invariant free-field equations of the form

$$
\left(\beta_{\mu} \partial^{\mu}+\operatorname{im}\right) \psi=0
$$

have been studied by many authors $[1-5,8,11-14]$ (see in particular Corson [13] and Takahashi [14] for complete bibliography). In the case that (1) yields solutions with only one mass (apart from sign), HarishChandra has shown [1] that the $\beta$-matrices must satisfy

$$
\left(\beta_{\mu} p^{\mu}\right)^{n}-p^{2}\left(\beta_{\mu} p^{\mu}\right)^{n-2}=0
$$

for some finite integer $n$. If one regards (2) as a polynomial in the variables $p_{0}, p_{1}, p_{2}$, and $p_{3}[12]$, the coefficients must vanish, and:

$$
\sum_{\substack{\text { permutations } \\\{1, \ldots, n\}}}\left(\beta_{\mu_{1}} \beta_{\mu_{2}}-g_{\mu_{1} \mu_{2}}\right) \beta_{\mu_{3}} \ldots \beta_{\mu_{n}}=0 .
$$

Umezawa and Visconti [3] claimed that (2) is satisfied for a minimum value of $n, n=2 s_{0}+1$, where $s_{0}$ is the maximum spin appearing in the representation of $S L(2, C)$ which acts on $\psi$ (we denote this representation by $S(\Lambda)$ ). This result, which seems to be generally accepted, is based on

* Part of this research was carried out at the University of British Columbia with the support of the National Research Council of Canada. 
the following facts, which are correctly treated in the above-mentioned paper.

Relativistic invariance of (1) requires that the L.H.S. of (2a) transform as a symmetric $n$-th rank Lorentz tensor under a similarity transformation involving $S^{-1}(\Lambda)$. If the L.H.S. of (2a) is denoted by $A_{\mu_{1} \mu_{2} \ldots \mu_{n}}$, then

$$
S^{-1}(\Lambda) A_{\mu_{1} \ldots \mu_{n}} S(\Lambda)=\prod_{i=1}^{n} \Lambda_{\mu_{i}}^{v_{i}} A_{v_{1} \ldots v_{n}} .
$$

If we consider only rotations, the L.H.S. of (3) cannot transform according to representations of $S U(2)$ which contain irreducible components of spin higher than $2 s_{0}$. Consequently, the R.H.S., regarded as an $n$-th rank symmetric Cartesian tensor with indices $\mu_{1} \ldots \mu_{n}$, must be reducible, in the event that $n>2 s_{0}$, to a symmetric tensor of rank $2 s_{0}$ or $2 s_{0}-1$ (as $n-2 s_{0}$ is even or odd, respectively) multiplied by the appropriate number of Kronecker $\delta$ 's. In any case, according to the standard procedures outlined by Weyl [6], $A_{\mu_{1} \ldots \mu_{n}}$ can be subdivided into terms which transform irreducibly under the Lorentz group, according to the representations $D^{(n / 2, n / 2)}, D^{(n / 2-1, n / 2-1)}, \ldots$, etc. According to Gårding's theorem [9], the parts of $A_{\mu_{1} \ldots \mu_{n}}$ which transform according to $D^{(k, k)}$, where $k>s_{0}$, must necessarily be trivial. Hence, if $n>2 s_{0}$,

$$
A_{\mu_{1} \ldots \mu_{n}}=\sum_{\text {perms. }} g_{\mu_{1} \mu_{2}} \ldots g_{\mu_{n-2 s_{0}-1} \mu_{n-2 s_{0}}} B_{\mu_{n-2 s_{0}+1} \ldots \mu_{n}}
$$

where $n-2 s_{0}$ is even, and

$$
A_{\mu_{1} \ldots \mu_{n}}=\sum_{\text {perms. }} g_{\mu_{1} \mu_{2}} \ldots g_{\mu_{n-2 s_{0}} \mu_{n-2 s_{0}+1}} B_{\mu_{n-2 s_{0}+2} \ldots \mu_{n}}
$$

where $n-2 s_{0}$ is odd. The tensor $B_{\mu_{1} \ldots \mu_{2}\left[n / 2-s_{0}\right]}$ is symmetric. The above can be more compactly expressed as

$$
A_{\mu_{1} \ldots \mu_{n}} \partial^{\mu_{1} \ldots} \partial^{\mu_{n}}=\sum_{\text {perms. }}\left(n-2 s_{0}\right) ! \square^{\left(\frac{n-2 s_{0}}{2}\right)} B_{\mu_{1} \ldots \mu_{2 s_{0}}} \partial^{\mu_{1}} \ldots \partial^{\mu_{2 s_{0}}}
$$

if $n-2 s_{0}$ is even, and

$A_{\mu_{1} \ldots \mu_{n}} \partial^{\mu_{1}} \ldots \partial^{\mu_{n}}=\sum_{\text {perms. }}\left(n-2 s_{0}+1\right) ! \square^{\left(\frac{n-2 s_{0}+1}{2}\right)} B_{\mu_{1} \ldots \mu_{2 s_{0}-1}} \partial^{\mu_{1}} \ldots \partial^{\mu_{2 s_{0}-1}}$

if $n-2 s_{0}$ is odd, where, now, $A_{\mu_{1} \ldots \mu_{n}} \partial^{\mu_{1}} \ldots \partial^{\mu_{n}}=n !(\beta \cdot \partial)^{n-2}\left((\beta \cdot \partial)^{2}-1\right)$.

The fallacy in existing discussions is to be found in the erroneous supposition that $B_{\mu_{1} \ldots \mu_{2 s_{0}}} \partial^{\mu_{1}} \ldots \partial^{\mu_{2 s_{0}}}$ is likewise a polynomial in $(\beta \cdot \partial)$. 
Indeed, in the special example of a spin $3 / 2$ equation included with this paper, the expression $\left((\beta \cdot \partial)^{2}-\square\right)(\beta \cdot \partial)^{2}$ reduces to $\square \cdot\left(\frac{1}{2}(\beta \cdot \partial) \beta_{\kappa} \beta^{\kappa}(\beta \cdot \partial)\right.$ $\left.-(\beta \cdot \partial)^{2}\right)$. In this example the form $(\beta \cdot \partial) \beta_{\kappa} \beta^{\kappa}(\beta \cdot \partial)$ is linearly independent from $(\beta \cdot \partial)^{2}, \beta_{\kappa}(\beta \cdot \partial)^{2} \beta^{\kappa}, \beta_{\kappa} \beta^{\kappa}(\beta \cdot \partial)^{2}$ and $(\beta \cdot \partial)^{2} \beta_{\kappa} \beta^{\kappa}$, and therefore cannot be reduced to an expression in $(\beta \cdot \partial)$ with scalar coefficients.

On the other hand, the formulae given for the differential operator $D(\partial)$ associated with the field commutation relations in the paper of Umezawa and Visconti [3] are quite correct. It is these formulae which lead to consideration of the tensor character of the expressions (2a).

The correct conclusion to be drawn from the preceding analysis is that the traceless part of any $n$-th rank symmetric tensor formed from the $\beta$ 's vanishes if $n>2 s_{0}$. As Weyl has shown [6], such a tensor must transform irreducibly under the action of the Lorentz group; in fact, it transforms according to the representation $D^{(n / 2, n / 2)}(\Lambda)$, which, as we have seen, is not non-trivially admissible. Moreover, as will be shown in a forthcoming paper, the same is true for any irreducible tensor classes constructed from the $\beta$ 's which correspond to representations of the Lorentz group that do not appear in the decomposition of $S(\Lambda) \times S(\Lambda)$ into a direct sum of irreducible representations [15]. The unique traceless part of the symmetric tensor $S_{\mu_{1} \ldots \mu_{n}}$ is given by:

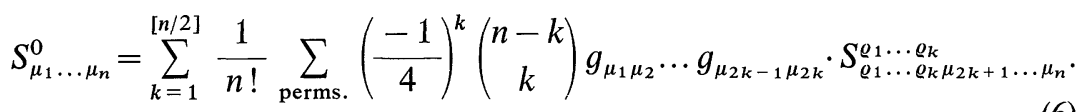

If we define the operator $\Pi_{\mu_{1} \ldots \mu_{n}}^{v_{1} \ldots v_{n}}$ as

$$
\begin{gathered}
\Pi_{\mu_{1} \ldots \mu_{n}}^{v_{1} \ldots v_{n}} \equiv \frac{1}{(n !)^{2}} \sum_{\substack{\text { perms. } \\
\{\mu\},\{v\}}} \sum_{k=1}^{[n / 2]}\left(\frac{-1}{4}\right)^{k}\left(\begin{array}{c}
n-k \\
k
\end{array}\right) \prod_{i=1}^{k} g_{\mu_{2 \imath-1} \mu_{2 \imath}} g^{v_{2 i-1} v_{2 i}} \\
\cdot \prod_{j=2 k+1}^{n} g_{\mu_{j}}^{v_{j}}
\end{gathered}
$$

we have $S_{\mu_{1} \ldots \mu_{n}}^{0}=\Pi_{\mu_{1} \ldots \mu_{n}}^{v_{1} \ldots v_{n}} S_{v_{1} \ldots v_{n}}$, and that $\Pi_{\mu_{1} \ldots \mu_{n}}^{v_{1} \ldots v_{n}} T_{v_{1} \ldots v_{n}}$, for an arbitrary tensor, $T_{\mu_{1} \ldots \mu_{n}}$, is the projection onto that part of itself which is symmetric and traceless. The projection operator $\Pi_{\mu_{1} \ldots \nu_{n}}^{v_{1} \ldots \nu_{n}}$ is analogous, but not equivalent, to the projection operator $\Theta_{\alpha_{1} \ldots \alpha_{n}}^{\beta_{1} \ldots \beta_{n}}$ constructed by Fronsdal $[7,10]$.

In considering the tensor $A_{\mu_{1} \ldots \mu_{n}}$, the term involving $g_{\mu_{1} \mu_{2}}$ has no traceless component. This follows from the fact that $g_{\mu v}$ is invariant under the Lorentz group, and any $n$-th rank tensor including it as a factor must transform in the manner of an $(n-2)$-th rank tensor. In the case of a symmetric tensor, $D^{(n / 2, n / 2)}(\Lambda)$ cannot then be included in the 
decomposition of the representation of the Lorentz group acting on that tensor into irreducible representations, whereas it is the representation which acts on the traceless part of an $n$-th rank symmetric tensor. The traceless part of the second term in (2a) is therefore identically zero. Accordingly,

so that

$$
\begin{aligned}
\Pi_{\mu_{1} \ldots \mu_{n}}^{v_{1} \ldots v_{n}} A_{v_{1} \ldots v_{n}} & \equiv \Pi_{\mu_{1} \ldots \mu_{n}}^{v_{1} \ldots v_{n}} \sum_{\text {perms. }}\left(\beta_{v_{1}} \ldots \beta_{v_{n}}-g_{v_{1} v_{2}} \beta_{v_{3}} \ldots \beta_{v_{n}}\right) \\
& =n ! \Pi_{\mu_{1} \ldots \mu_{n}}^{v_{1} \ldots v_{n}} \beta_{v_{1}} \ldots \beta_{v_{n}}
\end{aligned}
$$

$$
\Pi_{\mu_{1} \ldots \mu_{n}}^{v_{1} \ldots v_{n}} \beta_{v_{1}} \ldots \beta_{v_{n}}=0
$$

for $n>2 s_{0}$, which is the correct conclusion to be obtained from the hypotheses of the Umezawa-Visconti Theorem. It is, however, a weaker result than the one originally claimed. Eq. (8) expresses a relation between $n$-th rank, $(n-2)$-th rank, etc. symmetric tensors constructed from $\beta$-matrices. That this should lead to a polymonial equation satisfied by $p \cdot \beta$ ( $p$ a 4-vector) is only true if there exists a linear dependence among elements of the $\beta$-algebra such that tensors of the form

$$
\sum_{\text {perms. }} \beta_{\mu_{1}} \ldots \beta_{\mu_{n-2 k}}
$$

can be expressed in terms of tensors of the form

$$
g^{\mu_{1} \mu_{2}} \ldots g^{\mu_{2 p-1} \mu_{2 p}} \sum_{\text {perms. }} \beta_{\mu_{1}} \ldots \beta_{\mu_{n-2 k}}
$$

where $1 \leqq p \leqq \frac{n}{2}-k$, and contraction of tensor indices is implied. It should be realized that such a linear relation is an algebraic property of the $\beta$-matrices and cannot be inferred from their transformation properties under the Lorentz group.

\section{Appendix}

An Irreducible Single Mass, Single Spin Equation which Violates the Contention of Umezawa and Visconti

We define below the $\beta$-matrices for an irreducible spin $3 / 2$ Eq. (1) with unique mass and minimal equation for $\beta_{0}$ :

$$
\beta_{0}^{3}\left(\beta_{0}^{2}-1\right)=0 .
$$


We use the representation $S(\Lambda)=\left(D^{\left(1, \frac{1}{2}\right)} \oplus D^{\left(\frac{1}{2}, 1\right)}\right) \oplus 2\left(D^{\left(\frac{1}{2}, 0\right)} \oplus D^{\left(0, \frac{1}{2}\right)}\right)$. Denote the generators of $S(\Lambda)$ by $S_{\mu \nu}$ (antisymmetric tensor), with the identification $A_{i}=\frac{1}{2} \varepsilon_{i j k} S_{j k}$ and $B_{i}=S_{0 i}$.

$A_{i}$ and $B_{i}$ can be expressed as follows:

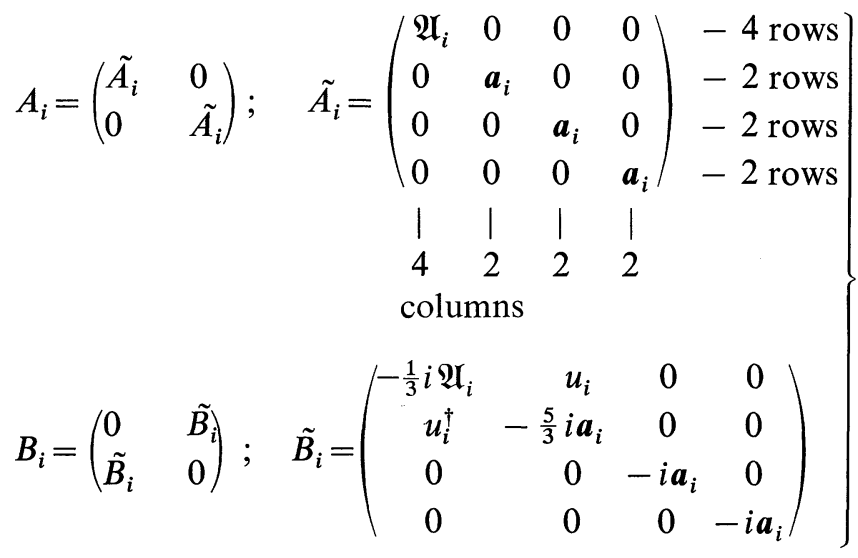

where

$$
\begin{aligned}
\mathfrak{I}_{1} & =-\frac{i}{2}\left(\begin{array}{rrrr}
0 & \sqrt{3} & 0 & 0 \\
\sqrt{3} & 0 & 2 & 0 \\
0 & 2 & 0 & \sqrt{3} \\
0 & 0 & \sqrt{3} & 0
\end{array}\right) ; \quad u_{1}=i \frac{\sqrt{2}}{3}\left(\begin{array}{rr}
\sqrt{3} & 0 \\
0 & 1 \\
-1 & 0 \\
0 & -\sqrt{3}
\end{array}\right) \\
\mathfrak{A}_{2} & =+\frac{1}{2}\left(\begin{array}{rrrr}
0 & -\sqrt{3} & 0 & 0 \\
\sqrt{3} & 0 & -2 & 0 \\
0 & 2 & 0 & -\sqrt{3} \\
0 & 0 & \sqrt{3} & 0
\end{array}\right) ; \quad u_{2}=+\frac{\sqrt{2}}{3}\left(\begin{array}{rr}
\sqrt{3} & 0 \\
0 & 1 \\
1 & 0 \\
0 & \sqrt{3}
\end{array}\right) \\
\mathfrak{U}_{3} & =-\frac{i}{2}\left(\begin{array}{rrrr}
3 & 0 & 0 & 0 \\
0 & 1 & 0 & 0 \\
0 & 0 & -1 & 0 \\
0 & 0 & 0 & -3
\end{array}\right) ; \quad u_{3}=-2 i \frac{\sqrt{2}}{3}\left(\begin{array}{rr}
0 & 0 \\
1 & 0 \\
0 & 1 \\
0 & 0
\end{array}\right) \\
\boldsymbol{a}_{1}= & -\frac{i}{2}\left(\begin{array}{ll}
0 & 1 \\
1 & 0
\end{array}\right) ; \quad a_{2}=\frac{1}{2}\left(\begin{array}{rr}
0 & -1 \\
1 & 0
\end{array}\right) ; \quad a_{3}=-\frac{i}{2}\left(\begin{array}{rr}
1 & 0 \\
0 & -1
\end{array}\right) .
\end{aligned}
$$

It can be seen that $\mathfrak{Q}_{i}=-i J_{i}^{(3 / 2)}$ and $\boldsymbol{a}_{i}=-\frac{i}{2} \sigma_{i}$, where $J_{i}^{(3 / 2)}$ are the usual operators associated with spin $3 / 2$ and $\sigma_{i}$ are the Pauli spin matrices. 
The following relations hold, where $[$,$] denotes the commutator and$ $\{$,$\} the anticommutator:$

$$
\left.\begin{array}{rl}
{\left[\mathfrak{I}_{i}, \mathfrak{A}_{j}\right]=\varepsilon_{i j k} \mathfrak{A}_{k} ; \quad\left[\boldsymbol{a}_{i}, \boldsymbol{a}_{j}\right]=\varepsilon_{i j k} \boldsymbol{a}_{k}} \\
\sum_{i=1}^{3} \mathfrak{U}_{i}^{2}=-\frac{15}{4} ; \quad \sum_{i=1}^{3} \boldsymbol{a}_{i}^{2}=-\frac{3}{4} ; \quad\left\{\boldsymbol{a}_{i}, \boldsymbol{a}_{j}\right\}=-\frac{1}{2} \delta_{i j}
\end{array}\right\}
$$

The remaining relations can be obtained by taking the hermitian conjugate of (10b), noticing that $\mathfrak{A}_{i}$ and $\boldsymbol{a}_{i}$ are antihermitian. From the above follow the usual commutation relations for $A_{i}$ and $B_{i}$ :

$$
\left[A_{i}, A_{j}\right]=\varepsilon_{i j k} A_{k} ; \quad\left[A_{i}, B_{j}\right]=\varepsilon_{i j k} B_{k} ; \quad\left[B_{i}, B_{j}\right]=-\varepsilon_{i j k} A_{k} .
$$

Relativistic invariance of (1) requires:

$$
\begin{aligned}
& {\left[\beta_{0}, A_{i}\right]=0 ; \quad\left[\beta_{0}, B_{i}\right]=\beta_{i} ;} \\
& {\left[\beta_{i}, A_{j}\right]=\varepsilon_{i j k} \beta_{k} ; \quad\left[\beta_{i}, B_{j}\right]=\delta_{i j} \beta_{0} .}
\end{aligned}
$$

Let

$$
S=\left(\begin{array}{rr}
\mathbf{1} & 0 \\
0 & -\mathbf{1}
\end{array}\right) ; \quad \eta=\left(\begin{array}{rr}
\tilde{\eta} & 0 \\
0 & -\tilde{\eta}
\end{array}\right) ; \quad \tilde{\eta}=\left(\begin{array}{rrrr}
1 & 0 & 0 & 0 \\
0 & 1 & 0 & 0 \\
0 & 0 & -1 & 0 \\
0 & 0 & 0 & -1
\end{array}\right) \begin{aligned}
& {[4 \text { rows }]} \\
& {[2]} \\
& {[2]} \\
& {[2]}
\end{aligned}
$$

Then $S$ is the reflection operator, since $\left[A_{i}, S\right]=0$ and $\left\{B_{i}, S\right\}=0$; in addition $\eta$ satisfies the requirements of a hermitizing matrix, since $\left(\eta A_{i}\right)^{\dagger}=-\eta A_{i},\left(\eta B_{i}\right)^{\dagger}=-\eta B_{i}, \eta=\eta^{\dagger}$, and $\eta^{2}=1$ (note that $A_{i}$ is antihermitian and $B_{i}$ is hermitian). 
The following vector set of matrices $\beta_{\mu}$ satisfies Lorentz-invariance of (1) and is hermitian with respect to $\eta$ (i.e. $\left.\left(\eta \beta_{\mu}\right)^{\dagger}=\eta \beta_{\mu}\right)$ :

$$
\begin{aligned}
& \beta_{0}=\left(\begin{array}{cc}
P_{0} & 0 \\
0 & -Q_{0}
\end{array}\right) ; \quad \beta_{i}=\left(\begin{array}{cc}
0 & P_{i} \\
-Q_{i} & 0
\end{array}\right) \\
& P_{0}=\left(\begin{array}{cccc}
1 & 0 & 0 & 0 \\
0-\frac{1}{2} & \frac{1}{2 \sqrt{3}} & \frac{\sqrt{2}}{\sqrt{3}} \\
0-\frac{1}{2 \sqrt{3}} & -\frac{1}{2} & 0 \\
0-\frac{\sqrt{2}}{\sqrt{3}} & 0 & 1
\end{array}\right) \\
& P_{i}=\left(\begin{array}{cccc}
-\frac{2}{3} i \mathfrak{U}_{i} & \frac{1}{2} u_{i} & -\frac{1}{2 \sqrt{3}} u_{i} & -\frac{\sqrt{2}}{\sqrt{3}} u_{i} \\
\frac{1}{2} u_{i}^{\dagger} & \frac{5}{3} i \boldsymbol{a}_{i} & \frac{i}{3 \sqrt{3}} \boldsymbol{a}_{i} & \frac{2}{3} i \frac{\sqrt{2}}{\sqrt{3}} \boldsymbol{a}_{i} \\
-\frac{1}{2 \sqrt{3}} u_{i}^{\dagger} & \frac{i}{3 \sqrt{3}} \boldsymbol{a}_{i} & i \boldsymbol{a}_{i} & 0 \\
-\frac{\sqrt{2}}{\sqrt{3}} u_{i}^{\dagger} & \frac{2}{3} i \frac{\sqrt{2}}{\sqrt{3}} \boldsymbol{a}_{i} & 0 & -2 i \boldsymbol{a}_{i}
\end{array}\right) \\
& Q_{\mu}=\tilde{\eta} P_{\mu} \tilde{\eta}, \quad \mu=0,1,2,3
\end{aligned}
$$

The elements written in $P_{0}$ stand for that number multiplying the appropriate identity matrix. Note than $P_{0}^{\dagger}=Q_{0}=\tilde{\eta} P_{0} \tilde{\eta}$, and $P_{i}^{\dagger}=P_{i}$.

It can be seen that the minimal equation satisfied by $\beta_{0}$ is $\beta_{0}^{5}-\beta_{0}^{3}=0$, which is of degree 1 greater than that normally predicted [3]. The only non-zero eigenvalues of $\beta_{0}$ are \pm 1 , which correspond to vectors which transform under rotations according to spin $3 / 2$ only. Thus the physical solutions have unique mass and spin.

Finally, the algebra formed from the $\beta$-matrices is irreducible. $\eta$ can be constructed from the $\beta$ 's, so that for every element $\mathfrak{a}$ in the algebra, its hermitian conjugate $\mathfrak{a}^{\dagger}$ is also in the algebra. Therefore, irreducibility of the algebra is equivalent to the requirement that no matrix commutes with all the $\beta$ 's, other than a multiple of the identity. The $S_{\mu \nu}$ can also be constructed from the $\beta$ 's, so that a commuting matrix $C$ would have to commute with the operators of the (complete) Lorentz group. The 
only such commutant must be of the form

$$
C=\left(\begin{array}{ll}
\tilde{C} & 0 \\
0 & \tilde{C}
\end{array}\right) ; \quad \tilde{C}=\left(\begin{array}{cccc}
\alpha & 0 & 0 & 0 \\
0 & \alpha & 0 & 0 \\
0 & 0 & a & b \\
0 & 0 & c & d
\end{array}\right) .
$$

To require that $\left[\beta_{0}, C\right]=0$ implies that $a=b=\alpha$ and $c=d=0$, which proves irreducibility. We append expressions for $\eta$ and $S_{\mu v}$.

$$
\begin{aligned}
\eta= & 9 \beta_{0}^{3}+3 \beta_{0}-\frac{3}{4} \beta_{\kappa} \beta_{0} \beta^{\kappa}-\frac{7}{4}\left\{\beta_{\kappa} \beta^{\kappa}, \beta_{0}\right\}-\frac{3}{4}\left\{\beta_{0}^{2}, \beta_{\kappa} \beta_{0} \beta^{\kappa}\right\} \\
& -\frac{3}{4} \beta_{0} \beta_{\kappa} \beta_{0} \beta^{\kappa} \beta_{0} .
\end{aligned}
$$

Let $\Pi_{0} \equiv \frac{3}{16} \beta_{\varrho} \beta_{\kappa} \beta^{\kappa} \beta^{\varrho}$ and $\Pi_{1} \equiv 1-\Pi_{0}$ : projections onto the subspaces acted on by $D^{\left(1, \frac{1}{2}\right)} \oplus D^{\left(\frac{1}{2}, 1\right)}$ and $2\left(D^{\left(\frac{1}{2}, 0\right)} \oplus D^{\left(0, \frac{1}{2}\right)}\right)$ respectively.

$$
\begin{aligned}
S_{\mu \nu}= & \Pi_{0}\left\{\frac{9}{8}\left(\beta_{\mu} \beta_{\kappa} \beta^{\kappa} \beta_{v}-\beta_{v} \beta_{\kappa} \beta^{\kappa} \beta_{\mu}\right)-2\left[\beta_{\mu}, \beta_{v}\right]\right\} \Pi_{0} \\
& +\Pi_{1}\left\{\frac{3}{2}\left[\beta_{\mu}, \beta_{v}\right]-\frac{9}{16}\left(\beta_{\mu} \beta_{\kappa} \beta^{\kappa} \beta_{v}-\beta_{v} \beta_{\kappa} \beta^{\kappa} \beta_{\mu}\right)\right. \\
& \left.-\frac{3}{16}\left\{\beta_{\varrho} \beta^{\varrho},\left[\beta_{\mu}, \beta_{v}\right]\right\}\right\} \Pi_{1} .
\end{aligned}
$$

Acknowledgement. The author wishes to express his sincere gratitude to Professors V. Bargmann and A. S. Wightman of Princeton University for their perceptive criticism and helpful advice during the preparation of this paper, and to the Mathematics Department of the University of British Columbia for their kind hospitality during the summer of 1970. He also wishes to thank Professor H. Umezawa of the University of Wisconsin for his comments on the manuscript.

\section{References}

1. Harish-Chandra: Phys. Rev. 71, 793 (1947).

2. - Proc. Roy. Soc. A 192, 195 (1947).

3. Umezawa, H., Visconti,A.: Nucl. Phys. 1, 348 (1956).

4. - Quantum field theory. Amsterdam: North Holland 1956.

5. Gel'fand, I. M., Minlos, R.A., Shapiro,Z.Ya.: Representations of the rotation and Lorentz groups and their applications. Oxford: Pergamon 1963.

6. Weyl, H.: The classical groups. Princeton: University Press 1946.

7. Behrends, R.E., Fronsdal, C.: Phys. Rev. 106, 345 (1957).

8. Capri, A.Z.: Phys. Rev. 178, 2427 (1969).

9. Gårding,L.: Kungl. Fysiograf Sällskap i Lund Förh. 13, 229 (1943).

- On a class of linear transformations connected with group representations (thesis, Medd. Lunds. Mat. Sem. 6, 1944).

10. Fronsdal, C.: Nuovo Cimento 9, 416 (1958). 
11. Bhabha, H. J.: Rev. Mod. Phys. 17, 200 (1945).

12. - Rev. Mod. Phys. 21, 451 (1949).

13. Corson, E. M.: Introduction to tensors, spinors, relativistic wave equations. London: Blackie 1953.

14. Takahashi, Y.: An introduction to field quantization. Oxford: Pergamon 1969.

15. Glass, A. S.: Lorentz-tensors and relativistic wave equations (thesis, Princeton University, 1971).

\author{
A. S. Glass \\ Eidg. Technische Hochschule Zürich \\ Seminar für Theoretische Physik \\ CH-8049 Zürich-Hönggerberg
}

